Revista Arbitrada Interdisciplinaria KOINONIA

Año 2020. Vol V. N${ }^{\circ}$. Especial: Contabilidad

Hecho el depósito de Ley: FA2016000010

ISSN: 2542-3088

FUNDACIÓN KOINONIA (F.K). Santa Ana de Coro. Venezuela.

Mayra Elizabeth Pinza-Sanmartín; Luis Ruben Guambaña-Cárdenas; Azucena Torres-Negrete

http://dx.doi.org/10.35381/r.k.v5i4.974

\title{
Aplicación de la NIC 12 impuesto a las ganancias en empresas camaroneras ecuatorianas
}

\section{Application of IAS 12 income tax in ecuadorian shrimp companies}

\author{
Mayra Elizabeth Pinza-Sanmartín \\ mayra.pinza06@est.ucacue.edu.ec \\ Universidad Católica de Cuenca, Cuenca \\ Ecuador \\ https://orcid.org/0000-0003-0699-3296 \\ Luis Ruben Guambaña-Cárdenas \\ Iguambana@ucacue.edu.ec \\ Universidad Católica de Cuenca, Cuenca \\ Ecuador \\ https://orcid.org/0000-0002-8031-3044 \\ Azucena Torres-Negrete \\ atorresn@ucacue.edu.ec \\ Universidad Católica de Cuenca, Cuenca \\ Ecuador \\ https://orcid.org/0000-0003-2538-8032
}

Recibido: 01 de junio de 2020

Revisado: 20 de junio de 2020

Aprobado: 02 de septiembre de 2020

Publicado: 24 de septiembre de 2020 


\title{
RESUMEN
}

El objetivo del presente estudio consistió en diseñar una guía metodológica para la aplicación de la NIC 12 en las empresas camaroneras, como mecanismo de determinación de resultados reales y base imponible de impuesta a la renta. De tipo descriptiva no experimental. Se determinó que la mayor parte de las empresas de camaroneras en la provincia de El Oro presentan sus estados financieros en forma anual, es así que, por lo que los profesionales contables valoran los activos biológicos al año y una cantidad minoritaria lo hacen de manera semestral (2,30\%), sin embargo, en algunos casos nunca han realizado valoraciones de sus activos biológicos como lo establece la normativa NIC 41. El proceso metodológico propuesto proporcionará una guía para la aplicación de la NIC 12, sustentada en la normativa tributaria vigente.

Descriptores: Tributación; impuesto sobre la renta; gasto; derecho laboral; gestión de personal. (Palabras tomadas del Tesauro UNESCO)

\begin{abstract}
The objective of this study was to design a methodological guide for the application of IAS 12 in shrimp companies, as a mechanism for determining real results and income tax base. Non-experimental descriptive type. It was determined that most of the shrimp companies in the province of El Oro present their financial statements on an annual basis, thus, therefore, accounting professionals value biological assets annually and a minority amount do so semi-annually $(2.30 \%)$, however, in some cases they have never carried out valuations of their biological assets as established by IAS 41 regulations. The proposed methodological process will provide a guide for the application of IAS 12, based on current tax regulations.
\end{abstract}

Descriptors: Taxation; income tax; expenditure; labour law; personnel management. (Words taken from UNESCO Thesaurus) 




Mayra Elizabeth Pinza-Sanmartín; Luis Ruben Guambaña-Cárdenas; Azucena Torres-Negrete

\section{INTRODUCCIÓN}

Las Normas Internacionales de Información Financiera (NIIF) son principios que se utilizan para la elaboración de los estados financieros, de esta manera se obtiene información financiera fiable, precisa y comprendida a nivel mundial; las cuales son emitidas por el Consejo de Normas Internacionales de Contabilidad, por tanto, la finalidad es garantizar que la información financiera proporcionada sea trasparente, precisa y comprensible con la finalidad de que los inversores y participantes de los mercados de capitales puedan tomar decisiones con base a información respaldada en políticas contables transparentes y consistentes (Brouwer \& Naarding, 2018).

Hoy en día, los estados financieros del sector camaronero no disponen de una estructura definida que estandarice los procedimientos y la aplicación correcta de las NIIF, así hacen referencia (Ruiz-Armijos, et al., 2019). Por consiguiente, si la siembra se produce en los últimos meses del año, esta deberá reflejarse como un ingreso, aunque no se realice sino el año siguiente y considerando la Norma Internacional de Contabilidad (NIC) 41, por tanto, la medición al valor razonable producirá una diferencia temporaria entre la base fiscal la base contable del ejercicio económico, sobre la cual se aplicará se aplicará la tasa de impuesto a la renta que corresponda y se registrará un pasivo por impuesto diferido que deberá ser pagado al momento que se lleve a cabo la venta del activo biológico.

Tomando en consideración el párrafo anterior, el impacto de la NIC 12 sería significativo al momento de presentar los estados financieros de las empresas del sector camaronero, ya que estas por ser empresas privadas generan gastos e ingresos de operaciones correspondientes al periodo que serán reconocidos, sin embargo, a la presente fecha todavía existen empresas camaroneras que no están aplicando al cierre del ejercicio contable la NIC 12, por consiguiente, se plantea como problema de investigación ¿cómo determinar el resultado real y la base imponible del impuesto a la renta al término del ejercicio económico en las empresas camaroneras?, por consiguiente, el objetivo del presente estudio consiste en diseñar una guía metodológica para la aplicación de la NIC 


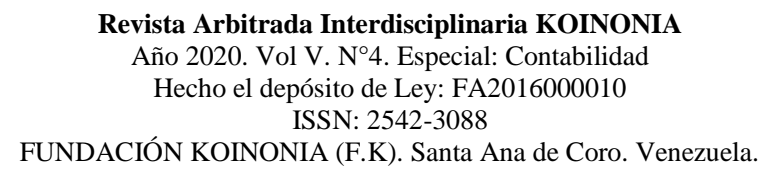

Mayra Elizabeth Pinza-Sanmartín; Luis Ruben Guambaña-Cárdenas; Azucena Torres-Negrete

12 en las empresas camaroneras, como mecanismo de determinación de resultados reales y base imponible de impuesta a la renta.

\section{Referencial teórico}

\section{Importancia de los principios contables en los estados financieros}

La globalización de la economía con la finalidad de incrementar sus ventajas competitivas y comparativas, ha impuesto diferentes retos a los países para que se mantengan y crezcan a nivel internacional, de esta manera, se ha buscado que algunos procesos mundiales sean sistematizados, uno de estos es la contabilidad, es así que, se asegura que las organizaciones a nivel mundial dispongan de un idioma financiero homogéneo y prácticas contables similares (Vargas \& Peña, 2017). De esta manera, surgen los principios contables que tienen la finalidad de formular criterios sobre la medición del patrimonio y la información de los elementos patrimoniales y económicos; y la gestión contable que hace referencia a la utilización, análisis e interpretación de la información financiera obtenida de la contabilidad de cara a la toma de decisiones (Quizhpi-Barbecho, et al., 2019).

La sostenibilidad de toda organización depende de la práctica de la contabilidad de gestión, por cuanto, el diagnóstico que se realice debe tener como base los principios contables (Oyewo, 2020). Es imprescindible aclarar que los principios hacen referencia a aspectos como el reconocimiento, medición, tratamiento, presentación y divulgación de servicios contables en el estado financiero (Tucker \& Scully, 2020); los mismos se desarrollaron de manera conjunta por el Instituto Americano de Contadores Públicos Certificados (AICPA) y el Instituto Colegiado de Contadores de Gestión (CIMA), tienen por finalidad vincular las prácticas contables en la creación de valor de las empresas a nivel global, identificando entre otros principios contables la objetividad, importancia relativa, revelación suficiente, comparabilidad, prudencia.

Las NIIF son principios que se utilizan en la práctica contable, de esta manera se establece información financiera fiable, precisa, comprendida a nivel mundial; 


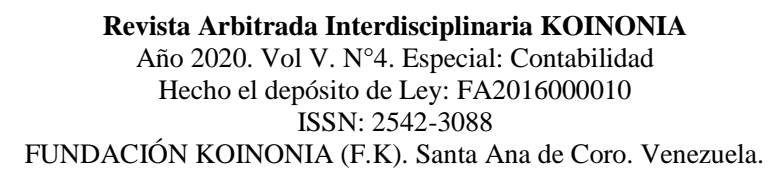

Mayra Elizabeth Pinza-Sanmartín; Luis Ruben Guambaña-Cárdenas; Azucena Torres-Negrete

conformadas por un manual que contiene las normas contables expuestas por el Consejo de Normas Internacionales de Contabilidad (IASB), que tiene su sede en Londres. La finalidad de las NIIF es armonizar la normativa contable a nivel internacional y garantizar que la información proporcionada sea trasparente, precisa y comprensible, para que los inversionistas y partícipes de capitales puedan tomar decisiones con base en información respaldada en políticas, principios contables y estándares técnicos (Brouwer \& Naarding, 2018).

Bajo el contexto mencionado, a partir del 01 de enero del año 2010, en el Ecuador y a causa de la globalización, se empezó a implementar las Normas Internacionales de Información Financiera (NIIF) de uso obligatorio según lo estable la Ley de Compañías (Cárdenas-Pañi, et al., 2020); la entidad a cargo de su reglamentación es la Superintendencia de Compañías (Asamblea Nacional del Ecuador, 2014). Como consecuencia de esto, se incluyó por primera vez el impuesto a las ganancias que hace mención a la sección 29 de la NIIF para PYMES y la NIC 12 (García \& Fonseca, 2017). Con base en lo expuesto, en el Ecuador, se realiza la conciliación entre la normativa contable NIC 12 y la normativa tributaria referida en la Ley de Régimen Tributario Interno (LRTI) la cual genera divergencias por el reconocimiento de impuestos corrientes y diferidos, que por un lado, deben ser registrados en la contabilidad, buscando revelar información financiera fiable; y por otro lado, deben ser analizados para establecer la base imponible del impuesto a la renta (Mena-Trujillo, et al., 2019). Por consiguiente, para las organizaciones comerciales es importante el hecho de reflejar los impuestos sobre la renta en los estados financieros, sin embargo, la falta de conformidad en las ganancias contables y fiscales conduce a la empresa a diferencias temporales e impuestos diferidos (Trofimova, et al., 2020). 


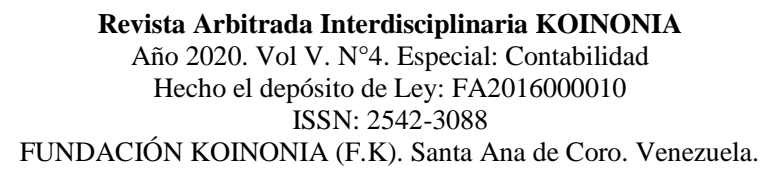

Mayra Elizabeth Pinza-Sanmartín; Luis Ruben Guambaña-Cárdenas; Azucena Torres-Negrete

\section{Los impuestos diferidos de acuerdo a la NIC 12 y su tratamiento contable}

Se considera como renta total los ingresos obtenidos sea de manera gratuita u onerosa por medio de actividades empresariales efectuadas por una persona natural, sucesión indivisa o sociedades nacionales o extranjeras domiciliadas en el Ecuador, así también, aquellos que se generen en el extranjero por personas naturales o sociedades que cuenten con domicilio en el país (Chávez-Cruz, et al., 2020). El impuesto a la renta es considerado como uno de los factores que influyen al momento de invertir en un país determinado, al respecto, las modificaciones hechas en América Latina en referencia a este impuesto se encaminan a gravar el gasto antes que la renta afectando de esta manera el ahorro y la inversión, esto ocasiona serias dificultades al hacer que su aplicación trascienda a las diferentes fuentes de ingresos del contribuyente (Brito-Gaona \& Iglesias, 2017). En este contexto, la NIC 12 impuesto a las ganancias ha tenido cambios desde el momento que fue publicada hasta la actualidad.

La NIC 12 presentó modificaciones en enero del año 2016, estos cambios tienen relación con el reconocimiento de activos de impuesto diferido por pérdidas no realizadas, dichas modificaciones entraron en vigencia a partir del 01 de enero del año 2017, su aplicación adelantada se consideró legal si las empresas revelaban y no limitaban los activos de impuestos diferidos, si clarificaban los principios generales a ser considerados. Cabe resaltar que los elementos de la NIC 12 no son evaluados de manera conjunta, con excepción de aquellos que tengan restricciones legales relacionadas con el uso de las pérdidas en las deducciones de algún impuesto en particular, situación que no ocurre en el Ecuador.

La aplicación de esta normativa es importante, ya que permite a las organizaciones hacer seguimiento a sus bases fiscales, además, les brinda información que servirá de utilidad al momento de tomar decisiones eficientes; este impuesto será aplicado poniendo atención al principio del devengado, considerando que es contabilizado bajo el enfoque del efecto impositivo (García \& Fonseca, 2017). Por tanto, los impuestos diferidos constituyen el valor que será pagado o recuperado en tiempo futuro y surgen de la 
aplicación de la base financiera al cierre de los estados financieros y la base tributaria aplicada en la determinación del impuesto a la renta del periodo contable. En los impuestos diferidos, acorde con la técnica contable y por efectos tributarios, solo se permite el reconocimiento de activos y pasivos en determinados casos y circunstancias, estas son:

1. Pérdidas por deterioro parcial como consecuencia del ajuste que se realiza con el fin de obtener el valor neto realizable del inventario, se considerarán como no deducibles con base al periodo contable inscrito; por este concepto se reconocerá un impuesto diferido, que se utiliza al momento de efectuarse la comercialización o consumo por los propietarios; en la actualidad, se considera como deducible y será presentado como impuesto diferido (Presidencia de la República, 2018).

2. En el caso de los contratos de construcción de uno o varios activos, si existe probabilidad de pérdidas, es decir, si los costos totales exceden a los ingresos totales que se espera obtener, se considerarán como no deducibles en el periodo en el que se hayan inscrito en la contabilidad, por este concepto, se procederá al reconocimiento de un impuesto diferido, se hace uso del impuesto diferido al momento que el contrato de la obra finalice, si la pérdida se produce.

3. La NIC 16 establece que el costo de un activo también incluirá los costos de desmantelamiento y retiro del elemento, además de la rehabilitación del lugar en el que se encuentra asentado el activo en cuestión, sin embargo, por fines de tributación, se considerará como no deducible en el período contable inscrito, por lo tanto, la depreciación concerniente al importe por desmantelamiento será reconocida como impuesto diferido, el que se utilizará al momento en que se haga efectiva esta actividad y en los casos en que exista obligación establecida para su ejecución.

4. El valor por deterioro de propiedades, planta y equipo, que sea utilizado en el proceso productivo, se considerará no deducible en el período contable inscrito, 




Mayra Elizabeth Pinza-Sanmartín; Luis Ruben Guambaña-Cárdenas; Azucena Torres-Negrete

como concepto de este será reconocido un impuesto diferido que se utilizará al momento de ser transferido el activo o su vida útil finalice.

5. Las provisiones diferentes a cuentas incobrables y desmantelamiento, se consideran no deducibles en el período contable inscrito y será reconocido un impuesto diferido, el cual podrá ser utilizado en el momento en que el contribuyente cancele la obligación por la cual se efectuó la provisión y hasta por el monto efectivamente pagado.

6. Las ganancias o pérdidas producto de la medición de activos no corrientes para ser comercializados, no es base para el impuesto a la renta en el período que se contabiliza, por este concepto será reconocido un impuesto diferido, el cual se utilizará cuando se produzca la venta o se haya pagado si su valoración ha generado ganancias, esto será si la venta corresponde a un ingreso gravado con impuesto a la renta.

7. Los activos biológicos que sean valorados con cambios en resultados en el transcurso de su transformación biológica y los ingresos y costos que se deriven de aplicar la normativa contable, serán tomados en cuenta en la conciliación tributaria como ingresos que no son sujetos de renta y costos atribuibles a ingresos no sujetos de renta; estas concepciones no serán consideradas al calcular la participación en trabajadores, gastos atribuibles para generar ingresos exentos, así como cualquier límite que establezca la norma tributaria que corresponda a estos elementos, por tanto, el impuesto a la renta se liquidará en el período en que se comercialice el activo biológico, para esto serán considerados los ingresos y costos reales acumulados que se le imputen a esta operación que permitirá determinar la base imponible

8. Las pérdidas declaradas después de la conciliación tributaria, correspondiente a ejercicios anteriores, con base en los términos y condiciones establecidas en la ley. 


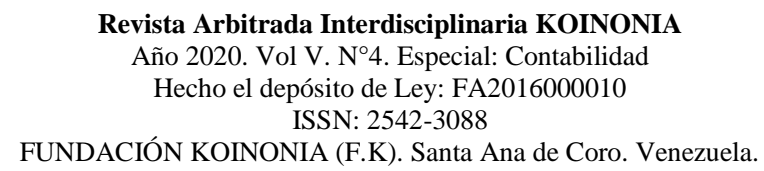

Mayra Elizabeth Pinza-Sanmartín; Luis Ruben Guambaña-Cárdenas; Azucena Torres-Negrete

9. Los créditos tributarios no utilizados, generados en operaciones anteriores, de acuerdo con los términos y condiciones establecidas en la ley.

10. En los contratos de servicios integrados con financiamiento de la contratista contemplados en la Ley de Hidrocarburos, las fórmulas de amortización previstas para fines tributarios deben ser compatibles con la técnica contable, el valor de la amortización de inversiones tangibles o intangibles registrado bajo la técnica contable que exceda al valor de la amortización tributaria de esas inversiones será considerado como no deducible en el periodo en el que se registre contablemente; sin embargo, se reconocerá un impuesto diferido por este concepto, durante los períodos en los cuales la amortización contable sea inferior a la amortización tributaria, según los términos establecidos en la Ley y en este Reglamento.

11. Las provisiones efectuadas para cubrir los pagos por desahucio y pensiones jubilares patronales constituidas a partir de la vigencia de la Ley Orgánica para la Reactivación de la Economía, Fortalecimiento de la Dolarización y Modernización de la Gestión Financiera no son deducibles, en este caso se reconocerá un impuesto diferido el cual será utilizado cuando se cancele la obligación por la que se efectuó la provisión y hasta por el monto efectivamente pagado.

12. Por el reconocimiento y medición de los ingresos, costos y gastos provenientes de contratos de construcción, con condiciones contractuales (Ibídem).

\section{Diferencias temporarias y permanentes en la determinación de la renta neta}

Los estados financieros tienen diferencias con las declaraciones fiscales, esto es común en la mayoría de países e incluso es aceptado por los estándares internacionales, que declaran a las diferencias como causantes del reconocimiento de impuestos diferidos, por ello se requiere tener una comprensión precisa de estos términos.

En este orden de ideas, se define a las diferencias temporarias como las discordancias existentes entre los montos inscritos en registros contables, sea de un activo o un pasivo y el importe que corresponde a la base fiscal y se clasifican en imponibles o deducibles. 




Mayra Elizabeth Pinza-Sanmartín; Luis Ruben Guambaña-Cárdenas; Azucena Torres-Negrete

Los impuestos diferidos tambien deben ser reconocidos en la pérdida fiscal que las empresas presentan en ejercicios fiscales anterioeres con la finalidad de ser compensada en los períodos siguientes, la cual no constaba reconocida en los estados financieros; las partidas que generan las diferencias temporarias son las que originan impuestos diferidos que es la diferencia entre el valor fiscal y su importe contable (Soto, et al., 2018).

En cuanto a las diferencias temporarias obligan a la empresa a ejecutar el ajuste del resultado en base de los registros contables y la declaración jurada, que consiste en una manifestación escrita o verbal de la veracidad de la información presentada (BelaundePlenge, 2018). Su origen está en los diferentes criterios temporarios de imputación utilizados con el fin de establecer la base imponible y el resultado contable antes de impuestos que se revertirán en periodos sucesivos, por consiguiente, las diferencias temporarias deducibles darán origen a cantidades que serán deducidas al momento en que se determine la ganancia o pérdida fiscal de ejercicios futuros, en el caso que el valor del activo existente en libros se haya recuperado o del pasivo liquidado (Soto, et al., 2018).

Por su parte, las diferencias permanentes se producen por determinación de la base imponible y el resultado contable, previo a la deducción de impuestos correspondientes al ejercicio, estos no se restituyen en otros períodos o ejercicios económicos y consecutivos, afectando de esta manera solo al ejercicio, pudiendo ser el ajuste positivo o negativo. En el momento en que existan discrepancias relacionadas con las políticas de reconocimiento deberán ser reveladas mediante las notas contables (Soto, et al., 2018).

Las diferencias que se presentan entre las bases tributarias y bases contables dan como resultado el registro de activos y/o pasivos por impuesto diferido, elemento importante para la toma de decisiones en la organización y para determinar el impacto ocasionado por el impuesto a las ganancias que genera alcances a diferentes proyectos financieros (Arreaga \& Salcedo, 2017). Con el propósito de que el activo o pasivo diferido se refleje de manera adecuada en los estados financieros, es imprescindible distinguir entre 


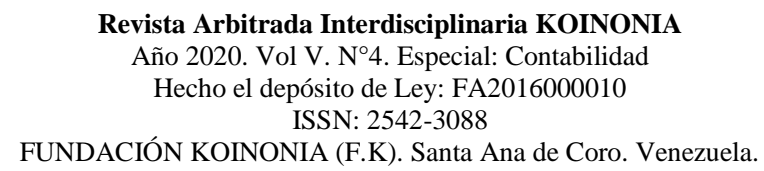

Mayra Elizabeth Pinza-Sanmartín; Luis Ruben Guambaña-Cárdenas; Azucena Torres-Negrete

diferencia temporaria y permanente, ya que estas surgen de operaciones que fiscalmente se consideran gastos deducibles o no deducibles, mientras que, en la contabilidad tienen otra interpretación.

En el caso de los activos, se medirán por el método del valor razonable definido en la NIIF 13, medición fundamentada en el mercado con la finalidad de estimar el precio de la transacción ordenada para la venta del activo, en la fecha establecida y en las condiciones de mercado vigentes y se clasifica de la siguiente manera:

a) Activo por impuesto diferido consiste en las cantidades de impuestos a la renta recuperables en ejercicios futuros a consecuencia de diferencias temporarias deducibles, créditos tributarios acumulados no utilizados, las pérdidas se compensarán en ejercicios posteriores, con un tiempo límite de 5 años como máximo (Paredes \& Deás-Albuerne, 2019); López-Balmaceda \& Soto-Guzmán, 2019).

b) Pasivo por impuesto diferido corresponde a los montos de impuesto a la renta a pagarse en ejercicios futuros que se relacionen con las diferencias temporarias imponibles; diferido se refiere a que las variaciones surgidas en el futuro supondrán un mayor pago de impuestos, por tanto, la cuenta utilizada es impuesto sobre los beneficios diferidos en aquellos casos en los que el ingreso no ha sido considerado por la parte fiscal, sin embargo, su característica es imponible, es decir que genera impuestos a la ganancia (Fierro, et al., 2016).

c) Para efectos tributarios se procederá al registro de activos y pasivos por impuestos diferidos en aquellos casos y condiciones que se encuentren establecidas en el reglamento; de existir divergencias entre las normativas tributarias y las normativas contables y financieras, serán las primeras las que predominen (Presidencia de la República, 2019).

Al existir diferencias en el tratamiento financiero, contable y tributario de determinadas partidas y para que puedan ser generados o recuperados a través de la conciliación tributaria, deben reflejarse en el estado de situación financiera en el importe correcto y 
con sus respectivos soportes, al respecto, en el Ecuador el 04 de diciembre del año 2015, se emitió la circular N NAC-DGECCGC15-00000012, que es la base legal para el tratamiento de los impuestos diferidos (Servicio de Rentas Internas, 2015).

De acuerdo con el artículo innumerado agregado a continuación del artículo 28 del registro oficial número 392 del Reglamento para la aplicación de la Ley Orgánica para el Fomento Productivo, Atracción de Inversiones, Generación de Empleo y Estabilidad y Equilibrio Fiscal, se realizan las siguientes reformas: los incisos primero y segundo presentes en el numeral 7 son sustituidos por los ingresos y costos que se derivan al momento de aplicar la norma contable correspondiente al reconocimiento y medición de los activos biológicos que sean medidos con cambios en los resultados en el transcurso de su período de transformación, serán considerados en conciliación tributaria como ingresos que no son sujetos de impuesto a la renta y costos que no son atribuibles a ingresos no sujetos de impuesto a la renta, incluso el pago pertinente a la participación a trabajadores, conceptos que no serán tomados en cuenta como gastos imputables a fin de generar ingresos independientes y en cualquier otro límite señalado en la norma tributaria.

La liquidación del impuesto a la renta se efectuará en el periodo fiscal en el que se haya producido la comercialización o distribución del activo biológico, se tomará en consideración aquellos ingresos que se le atribuyan al reconocimiento y medición de activos biológicos y los concernientes a la venta o disposición del activo y los costos acumulados que se le imputen a la operación, además de esto, también se considerará la participación de utilidades para los trabajadores cancelada a la fecha de la operación que determina la base imponible (Presidencia de la República, 2018). 


\section{MÉTODO}

El presente artículo se fundamentó en un diseño de investigación no experimental y transversal, puesto que la recolección de información y su análisis se desarrolló en un momento único en el tiempo, las variables fueron observadas y analizadas sin manipularlas de manera intencional; el alcance de esta investigación fue descriptivo, porque se analizó la Norma Internacional de Contabilidad 12 en las empresas del sector camaronero y la base fiscal en los estados financieros, para llegar así a conclusiones generales concernientes a los impuestos diferidos y la determinación de la base imponible para el cálculo del impuesto a la renta en el sector camaronero.

El universo de estudio de la presente investigación fue de 522 empresas del sector camaronero inscritas en la Superintendencia de Compañías Valores y Seguros (SCVS), la técnica empleada para la recopilación de información fue la encuesta, se utilizó el método de muestreo probabilístico-aleatorio simple, obteniendo una muestra de estudio de 220 empresas, la información recopilada fue procesada en función de la estadística descriptiva.

\section{RESULTADOS}

Presentación de estados financieros para el cálculo del impuesto a la renta: los estados financieros elaborados bajo estándares internacionales constituyen el insumo base para la elaboración de la conciliación tributaria y esta a su vez mediante los ajustes establecidos en la normativa tributaria permiten la determinación de la base imponible para el cálculo del impuesto a la renta. Al respecto, el 41,10\% de encuestados afirman que presentan sus estados financieros considerando estándares internacionales, mientras que, el $58.90 \%$ no aplican las NIIF, este resultado determina que gran parte de empresas camaroneras no cuentan con información financiera comparable y transparente, por lo tanto, el cálculo de la base imponible para la determinación del impuesto a la renta a pagar, tiene errores. 


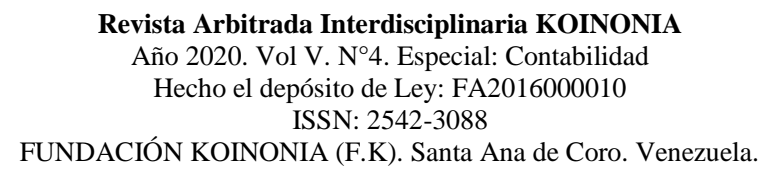

Mayra Elizabeth Pinza-Sanmartín; Luis Ruben Guambaña-Cárdenas; Azucena Torres-Negrete

Capacitación: la falta de capacitación y actualización del personal contable sobre reformas contables y tributarias genera información inconsistente y no fiable, en este sentido, el $79,80 \%$ de encuestados afirman que han participado en procesos de capacitación y actualización en normas contables y tributarias.

Valorización de activos biológicos: consiste en actualizar el valor del activo biológico al valor del mercado activo, permite reflejar el valor actual de los activos de la empresa y proporciona información necesaria para los accionistas y entidades financieras que requieran hacer evaluación de los estados financieros, de acuerdo con la encuesta aplicada el $70,80 \%$ de los contadores señalan que no realizan mediciones para valorar los activos biológicos, y tan solo, el $29.20 \%$ de los encuestados realizan la valoración de los activos biológicos al término del ejercicio fiscal. Al no aplicar la NIC 41 en la valoración anual del activo biológico se está presentando información errónea que afecta a la base para el cálculo del impuesto a la renta.

Participación de utilidades para trabajadores: la valorización del activo biológico no se considera para la base de cálculo de la participación laboral atribuible que se refleja en el estado de resultados y obligaciones laborales del contribuyente, en consecuencia, se generan diferencias considerables en el balance, estado de resultados y el flujo de efectivo. Con respecto a este tema, el 88,40\% afirman que la reforma al artículo 28 de la LRTI no genera ningún impacto en el rubro de participación a trabajadores, en tal sentido, desconocen su aplicación, mientras que, el 7,30\% de los contadores, evidencia que si afecta.

Impuestos diferidos: las NIIF prescriben que todos los activos y pasivos reconocidos en el estado de situación financiera de una empresa se deben determinar el reconocimiento de activos y pasivos, al respecto, el $28,60 \%$ mencionaron que si aplican el reconocimiento de impuestos diferidos, mientras que, $71,40 \%$ no realizan reconocimiento de activos y pasivos por impuestos diferidos al cierre del ejercicio fiscal, como consecuencia de este resultado, se deduce que un importante número de empresas perderán la posibilidad de 
recuperar alguna partida que en un año fue considerado no deducible hasta que está se realice.

\section{PROPUESTA}

Con base en los resultados obtenidos se plantea una guía metodológica para el tratamiento del impuesto a las ganancias al final del ejercicio económico en el sector camaronero (ver figura 1).



Figura 1. Esquema de una guía metodológica del tratamiento del impuesto a las ganancias.

\section{Descripción de la metodología}

La conciliación tributaria surge como producto de las diferencias existentes entre los resultados contable y fiscal, esta será realizada solo al momento de declarar el impuesto a la renta por las organizaciones y personas naturales que tengan obligación de llevar contabilidad. 


\section{Determinación de diferencias temporarias}

Las diferencias temporarias son aquellas que se producen en el momento en que no existe coincidencia entre los importes que se imputan al beneficio contable y a la base imponible, positivas o negativas, tienen origen en un periodo y desaparecen en otro.

\section{Base tributaria fuente}

Para el cálculo del impuesto a la renta es necesario establecer la base imponible. Una vez finalizado el ejercicio económico anual, se procede a la conciliación entre las bases contable y fiscal, comparación que se desarrolla por medio del método del pasivo basado en el balance (MPBB) y se contabiliza el efecto impositivo:

a) Las diferencias temporarias imponibles 0 deducibles se generan como consecuencia de las diferencias entre la base fiscal y el valor en libros de los activos y pasivos.

b) Pasivos y activos por impuestos diferidos se establecen mediante la aplicación de la tasa impositivo a las diferencias temporarias.

c) El origen de la contabilización de los pasivos y activos por impuestos diferido es la base fiscal.

\section{Análisis contable de la incidencia de la aplicación de los impuestos diferidos}

\section{Activos}

Diferencias permanentes: las diferencias permanentes constituyen aquellos gastos o ingresos contables que son consideradas para determinar la base imponible de acuerdo a la ley, estos no serán posibles de superar en el tiempo, esto ocasionará modificaciones en el resultado contable, por el aumento en el impuesto a la renta causado.

Las diferencias permanentes positivas se producen al momento que el gasto contable no es deducible de manera fiscal, mientras que en las negativas el ingreso contable no tributa. 
Diferencias temporarias imponibles y deducibles: las diferencias temporarias imponibles implican el reconocimiento de pasivos por impuestos diferidos, a estos se le aplicará la tarifa del impuesto a la renta que corresponde de acuerdo a la ley, es decir, representan importes a ser pagados en periodos futuros los deducibles generarán mayores cantidades a cancelar las cuales serán devueltas mediante en ejercicios futuros.

\section{Valor Neto de realización}

La Compañía XXXX, vende insumos para camarón de los cuales se ha determinado que existe al 31 de diciembre del 2019, una pérdida por Medición de Valor Neto de Realización, las cuales se presenta a continuación, (figura 2):

\begin{tabular}{|l|r|} 
& $\begin{array}{l}\text { Valor del } \\
\text { Costo unitario }\end{array}$ \\
\hline & $\begin{array}{r}\text { del Producto } \\
\text { Insumos }\end{array}$ \\
\hline Vitamina C 1KG & $15 / 2020$ \\
\hline Oxi ric 25KG & 2,00 \\
\hline Ferti Humico 25Kg & 6,00 \\
\hline Aquablend c & 5,00 \\
\hline BioBac A 5ltros & 6,00 \\
\hline Fordex 20Kg & 15,00 \\
\hline Silicato 25Kl & 25,00 \\
\hline Nuttrilake 25g & 12,00 \\
\hline & 86,00 \\
\hline
\end{tabular}

\begin{tabular}{|c|c|c|}
\hline \multicolumn{3}{|c|}{ Medición por unidad } \\
\hline$\frac{\begin{array}{c}\text { Valor en el } \\
\text { Mercado al } 31\end{array}}{\underline{\text { de diciembre }}}$ & $\frac{\frac{(-) \text { Costo }}{\text { asociados a su }}}{\text { venta }}$ & $\begin{array}{l}\frac{(=) \text { Valor }}{\text { Neto de }} \\
\underline{\text { Realización }}\end{array}$ \\
\hline 12,00 & $-1,15$ & 10,85 \\
\hline 1,80 & $-0,52$ & 1,28 \\
\hline 5,40 & $-1,20$ & 4,20 \\
\hline 4,00 & $-1,23$ & 2,77 \\
\hline 5,80 & $-1,50$ & 4,30 \\
\hline 13,00 & $-1,23$ & 11,77 \\
\hline 22,00 & 1,23 & 23,23 \\
\hline 10,00 & $-1,10$ & 8,90 \\
\hline 74,00 & $-6,70$ & 67,30 \\
\hline
\end{tabular}

\begin{tabular}{|c|c|c|}
\hline$\frac{\begin{array}{c}\text { Ajustes por } \\
\text { Medición de VNR, }\end{array}}{\text { por unidad }}$ & $\begin{array}{l}\underline{\text { Unidades }} \\
\text { mantenidas } \\
\underline{\text { en STOCK }}\end{array}$ & $\begin{array}{l}\frac{\text { Ajustes por }}{\text { Medición de }} \\
\underline{\text { VNR, Global }}\end{array}$ \\
\hline 4,15 & 120 & 498 \\
\hline 0,72 & 90 & 64,8 \\
\hline 1,80 & 110 & 198 \\
\hline 2,23 & 120 & 267,6 \\
\hline 1,70 & 300 & 510 \\
\hline 3,23 & 125 & 403,75 \\
\hline 1,77 & 152 & 269,04 \\
\hline 3,10 & 320 & 992 \\
\hline 18,70 & $1.337,00$ & $3.203,19$ \\
\hline
\end{tabular}

Figura 2. Estimación del valor neto de realización de inventario 
En el análisis realizado por la compañía, se determinó un ajuste por valor neto de realización de US\$3.203.19, los cuales se deben ajustar conforme al siguiente asiento de diario (figura 3):

\begin{tabular}{|lccc|}
\hline RUBRO & CONCEPTO & Debe & Haber \\
\hline Gastos & Pérdida Neta por Deterioro en el Valor de inventario & $3.203,19$ & \\
\hline Activo & (-) Estimación Valor Neto de Realización Inventarios & & $3.203,19$ \\
\hline P/R La estimación del valor neto de realización de inventarios & & \\
\hline
\end{tabular}

Figura 3. Asiento contable de estimación del valor neto de realización de inventario

El gasto reconocido en el asiento expuesto para efectos tributario, se debe considerar como no deducible, generando una diferencia temporaria, entre sus bases contables y tributarias, el cual se deducirá en el momento de la venta de los bienes, a continuación, se detalla lo mencionado en el Reglamento a la Ley de Régimen Tributario Interno en el numeral 1 del artículo innumerado a continuación del Art. 28: Por lo cual la Compañía debe registrar el reconocimiento de un activo por impuesto diferido, el cual se muestra a continuación (figura 4):

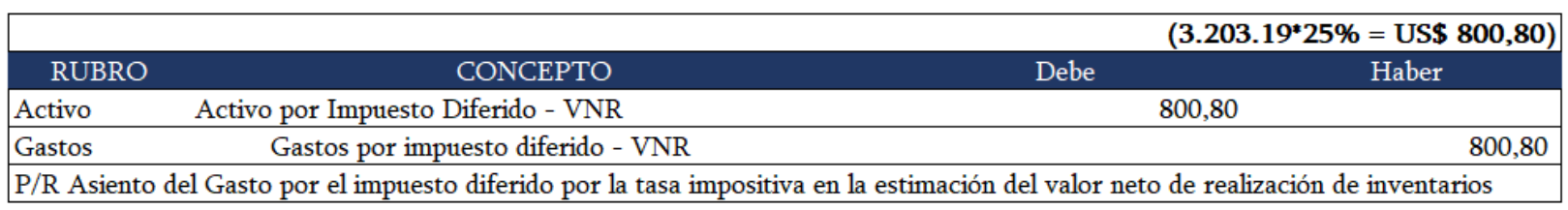

Figura 4. Asiento contable por el impuesto diferido al aplicar la tasa impositiva en la estimación del valor neto de realización de inventario.

\section{Incidencia de la NIC 12 en la valorización de activos biológicos}

La valoración del activo biológico se realiza considerando la suma de los costos de larva, balanceado, mano de obra, vitaminas y otros que intervengan en el crecimiento del camarón y se compara con el valor presente del mercado, se reconocer un ingreso por su crecimiento al cierre de cada ejercicio, tomando en referencia cuando tiene la talla de 8 gramos o superior, asumiendo el precio de mercado se solicita vía mail una o varias 
cotizaciones a las exportadoras de camarón, para el precio de mercado activo las exportadoras de camarón toman en cuenta en las cotizaciones la textura y talla que se obtiene del histograma de la piscina enviado por el biólogo de la camaronera, con el precio de mercado obtenido menos los costos de producción se contabiliza el asiento de ajuste por valor razonable (ver figura 5).

\section{Contabilización del cultivo de camarón-valor razonable}

\section{Piscina de camarón con gramaje $>=8$ gramos} Activos biológicos-sin valoración

\begin{tabular}{|l|l|r|}
\hline Activo & Larva & $3.625,00$ \\
\hline Activo & Balanceado & $1.585,00$ \\
\hline Activo & Mano de Obra & $8.965,00$ \\
\hline Activo & Vitamina & $1.686,00$ \\
\hline \multicolumn{2}{|c|}{ total: } & $15.861,00$ \\
\hline
\end{tabular}

\begin{tabular}{|l|l|r|}
\multicolumn{3}{|c|}{ Activos Biológicos-con valoración } \\
\hline Activo & Larva & $3.625,00$ \\
\hline Activo & Balanceado & $1.585,00$ \\
\hline Activo & Mano de Obra & $8.965,00$ \\
\hline Activo & Vitamina & $1.686,00$ \\
\hline & Ajustes por Valor & \\
Activo & Razonable & $5.422,86$ \\
\hline \multicolumn{2}{|l|}{ total: } & $21.283,86$ \\
\hline
\end{tabular}

Valor razonable-precio de mercado $21.283,86$

Precio de activo biológico sin valoración

Ajuste a realizar

Figura 5. Valoración razonable

Corresponde al ingreso que se genera por el crecimiento del camarón, el cual debe ser reconocido como ingresos en el ejercicio 2019, a continuación, se presenta el registro correspondiente (figura 6):

\begin{tabular}{|lll|}
\hline \multicolumn{1}{|c|}{ Reconocimiento del Ingreso } & DEBE & HABER \\
\hline Activo Biologico Medidos a valor Razonable & $5.422,86$ & \\
\hline $\begin{array}{l}\text { Ganancias netas por mediciones de activos biológicos a valor razonable menos } \\
\text { costos de venta }\end{array}$ & $5.422,86$ \\
\hline P/R Asiento de las ganancias por medición de activo biológico a valor razonable. & \\
\hline
\end{tabular}

Figura 6. Asiento contable de las ganancias en medición de activo biológico. 
El reconocimiento de la ganancia por medición de activos biológicos, a valor razonable menos costo de venta, no es sujeto a impuesto a la renta, generándose una diferencia temporaria entre su base fiscal y contable, tal como lo menciona el Reglamento a la Ley de Régimen Tributario en el numeral 7 del artículo innumerado a continuación del Art. 28. En el período fiscal en el que se produzca la venta o disposición del activo biológico, se efectuará la liquidación del impuesto a la renta, para lo cual se deberán considerar los ingresos atribuibles al reconocimiento y medición de activos biológicos y aquellos relacionados con la venta o disposición del activo y los costos reales acumulados imputables a dicha operación, así como la participación laboral atribuible y pagada a la fecha de la operación, para efectos de la determinación de la base imponible."

En este sentido, se debe reconocer un pasivo por impuesto diferido, cabe mencionar que para el ejercicio analizado se ha asumido que la tasa impositiva de la compañía es del $25 \%$, por tanto, el reconocimiento del pasivo por impuesto diferido se reconoce y se exponen a continuación (figura 7):

\begin{tabular}{|c|c|c|}
\hline \multicolumn{3}{|c|}{$($ US\$5.422,86*25\%) = US\$ $1.355,72$} \\
\hline & DEBE & HABER \\
\hline Gasto por impuesto a la renta diferido & 1.355 & \\
\hline Impuesto Diferido Activo Biologico & & $1.355,72$ \\
\hline
\end{tabular}

Figura 7. Asiento contable del impuesto diferido por la tasa impositiva de las ganancias en medición de activo biológico

\section{Análisis del impacto tributario en resultados}

En el cierre del ejercicio comparativo del año fiscal 2019 se registra el total de la utilidad incluida la valoración, la reversión de la valoración del año anterior en positivo y en negativo, como la normativa tributaria contempla que no se adiciona la valoración de activos biológicos del año presente para la base imponible antes de participación trabajadores, en este cálculo, se procede a aplicar el 15\% de participación a trabajadores 
Revista Arbitrada Interdisciplinaria KOINONIA

Año 2020. Vol V. N ${ }^{\circ}$. Especial: Contabilidad

Hecho el depósito de Ley: FA2016000010

ISSN: 2542-3088

FUNDACIÓN KOINONIA (F.K). Santa Ana de Coro. Venezuela.

Mayra Elizabeth Pinza-Sanmartín; Luis Ruben Guambaña-Cárdenas; Azucena Torres-Negrete

permitiendo observar un incremento en su valor, adicional se realiza el asiento contable del gasto por impuesto diferido, multiplicando por el $25 \%$ del impuesto a la renta, (ver figura 8) se exponen a continuación:

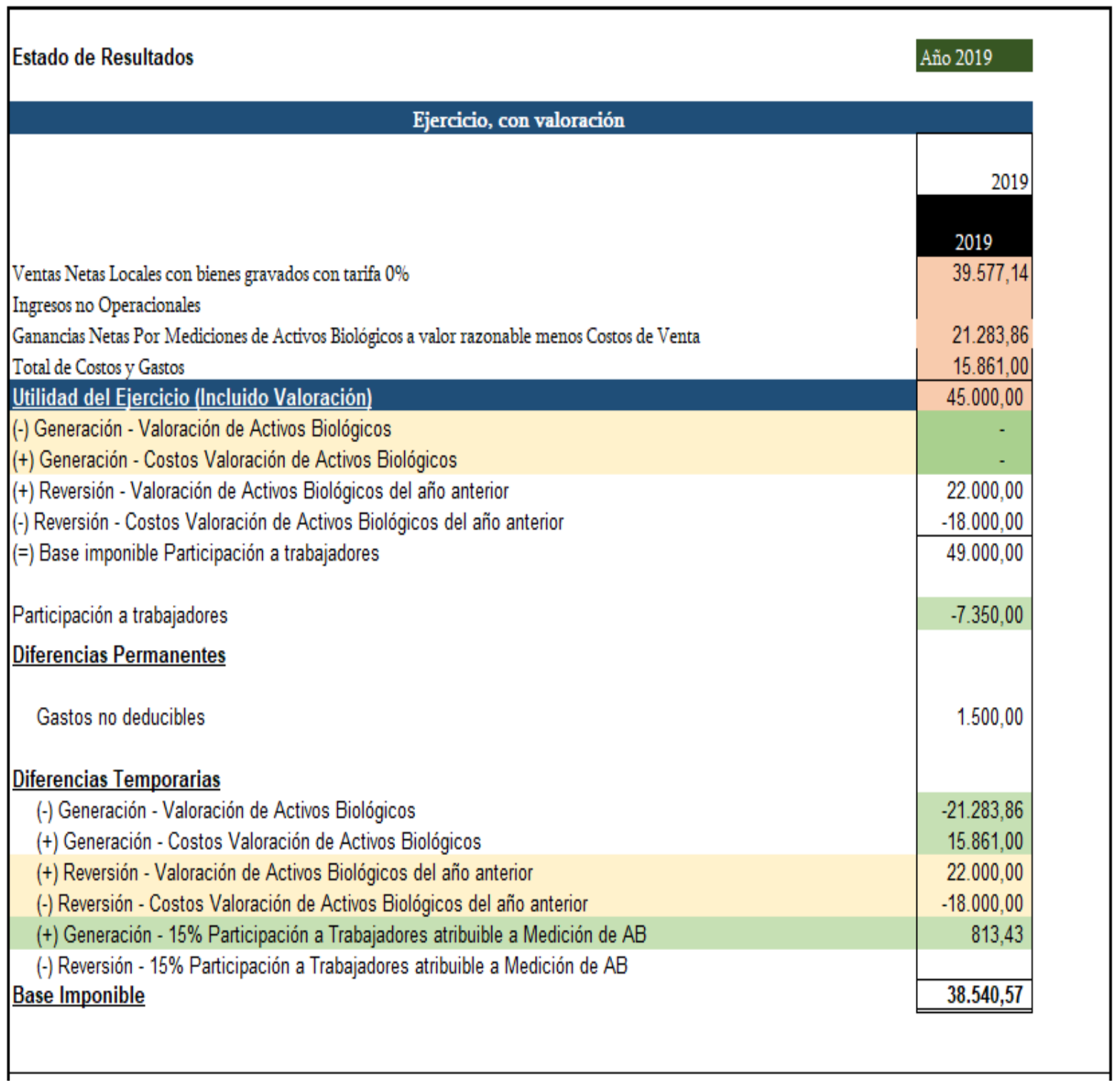

Figura 8. Estado de resultados. 
Corresponde a la ganancia, por la medición del activo biológico, la cual se resta en la conciliación tributaria (diferencia temporaria), cabe mencionar que para efecto de presentación del formulario 101 se divide en valoración de activos biológicos menos su costo correspondiente, dando como resultado el ingreso por ganancia a valor razonable. La conciliación tributaria establece en el ejemplo una utilidad de 45.000,00 incluida la valoración de activos biológicos por $21.283,36$, se agrega la reversión por ingresos y costos del año 2018, por lo que se genera la base de cálculo de participación a trabajadores de $49.000,00$, se aplica el $15 \%$ a fin de obtener la participación. A la base antes mencionada se les resta la participación a trabajadores, se resta el resultado entre los ingresos por generación y los gastos por diferidos, se adiciona los gastos no deducibles y se genera la base del impuesto a la renta con una tarifa impositiva del $25 \%$. (ver figura 10), se exponen a continuación:



Figura 9. Conciliación tributaria. 
Según el Reglamento de la Ley de Régimen Tributario Interno, el 15\% calculado de participación a trabajadores, atribuible a la ganancia por medición de valor razonable (US\$ 5,422.86 ${ }^{*} 15 \%=$ US\$813.43), debe ser considerada como no deducible en el ejercicio que se origina, y se utilizará en el periodo que se venda el activo biológico (camarón), lo cual da origen a una nueva diferencia temporaria, $813.43 * 25 \%=203.36$, la misma se registra de la siguiente manera (figura 10):

\begin{tabular}{|c|c|c|}
\hline Asientos de Diarios & Debe & Haber \\
\hline Activo por impuesto diferido PT & 203,36 & \\
\hline Gastos por Impuesto Diferido & & 203,36 \\
\hline
\end{tabular}

Figura 10: Asiento contable de activo diferido por la tasa impositiva por participación de utilidades.

\section{CONCLUSION}

Los países a nivel mundial han optado por la aplicación de las Normas Internacionales de Contabilidad, ya que permite disponer de estados financieros con información fiable, comprensible y comparable a nivel internacional, además su adecuada adopción permite revelar la realidad económica y financiera de las operaciones, lo cual contribuye para la toma de decisiones.

La adopción de estas normativas ha evidenciado beneficios y obstáculos en diferentes países del mundo, efectos financieros que dependen de circunstancias particulares, sus políticas contables y la precisión de sus estimaciones; en este contexto, la NIC 12 posibilita el tratamiento contable del impuesto a las ganancias, conocido en Ecuador como impuesto a la renta tomando en consideración las consecuencias presentes y 
futuras de la recuperación o liquidación de activos y pasivos y de las transacciones y otros eventos del periodo corriente que han sido reconocidos en los estados financieros. Entre los principales resultados del diagnóstico realizado en la investigación se determinó que la mayor parte de las empresas de camaroneras en la provincia de El Oro presentan sus estados financieros en forma anual, es así que, por lo que los profesionales contables valoran los activos biológicos al año y una cantidad minoritaria lo hacen de manera semestral $(2,30 \%)$, sin embargo en algunos casos nunca han realizado valoraciones de sus activos biológicos como lo establece la normativa NIC 41 .

La reforma al artículo innumerado agregado a continuación del artículo 28 del Reglamento para la aplicación de la Ley Orgánica para el Fomento Productivo, considera un incremento en la participación laboral atribuible que se refleja en el estado de resultados, como consecuencia se generan diferencias considerables en el balance, estado de resultados y el flujo de efectivo, las mismas que deben ser valoradas en el sector camaronero.

El proceso metodológico propuesto proporcionará una guía para la aplicación de la NIC 12, sustentada en la normativa tributaria vigente de tal forma que se garantice la determinación de resultados reales en el sector camaronero, además el procedimiento explica cómo se determinarán las diferencias temporales en los estados financieros a través de la comparación de la cuantía en libros de los activos y pasivos en correspondencia con la base fiscal conveniente.

\section{FINANCIAMIENTO}

No monetario.

\section{AGRADECIMIENTO}

A las empresas de camaroneras en la provincia de El Oro; por apoyar el desarrollo de la investigación. 


\section{REFERENCIAS CONSULTADAS}

Arreaga, D., \& Salcedo, R. (2017). NIC 12 y la Relación en el Cálculo del Impuesto a la Renta: Caso Patel Exports S.A., Patexports Guayaquil Ecuador. [IAS 12 and the Relationship in the Calculation of Income Tax: Case of Patel Exports S.A., Patexports Guayaquil Ecuador]. Revista Observatorio de la Economía Latinoamericana, Ecuador, 1(1), 1 - 12. Recuperado de https://cutt.ly/MiQyW4e

Asamblea Nacional del Ecuador. (2014). Ley de compañías. [Company law]. Recuperado de https://cutt.ly/liQecnU

Belaunde-Plenge, W. (2018). Efecto de los cambios en las normas contables en la tributación empresarial. [Effect of changes in accounting standards on corporate taxation]. Derecho \& Sociedad, (50), 157-169.

Brito-Gaona, L, \& Iglesias, E. (2017). Inversión privada, gasto público y presión tributaria en América Latina. [Private investment, government expenditure and tax burden in Latin America]. Estudios de economía, 44(2), 131156. https://dx.doi.org/10.4067/S0718-52862017000200131

Brouwer, A., \& Naarding, E. (2018). Making Deferred Taxes Relevant. Accounting in Europe, 15(2), 200-230. https://doi.org/10.1080/17449480.2018.1451903

Cárdenas-Pañi, M., Narváez-Zurita, C., Erazo-Álvarez, J., \& Torres-Palacios, M. (2020). Conciliación de impuestos a las ganancias: Un estudio bajo la normativa fiscal y normativa contable. [Reconciliation of income taxes: A study under tax regulations and accounting regulations]. Revista Arbitrada Interdisciplinaria Koinonía, 5(10), 757-773. http://dx.doi.org/10.35381/r.k.v5i10.715

Chávez-Cruz, G, Chávez-Cruz, R, \& Betancourt-Gonzaga, V. (2020). Análisis de la contribución del IVA, renta, Rise e Ice en la zona 7 del Ecuador periodo 2013-2017. [Analysis of the contribution of VAT, income, Rise and Ice in area 7 of Ecuador period 2013-2017]. Revista Universidad y Sociedad, 12(2), 330-335.

Fierro, Á., Fierro, F., \& Fierro, F. (2016). Contabilidad de pasivos con estándares internacionales para pymes. [Liability accounting with international standards for SMEs] (3ra edición ed.). Bogotá: Ecoe ediciones.

García, G., \& Fonseca, M. (2017). Impuesto a las ganancias. Un estudio del costo beneficio de su aplicación en las PYMES del Ecuador. [income tax. A study of the cost benefit of its application in SMEs in Ecuador]. Actualidad Contable Faces, 20(35), 26-45. 
López-Balmaceda, T., \& Soto-Guzmán, F. (2019). Cómo revelan las sociedades anónimas abiertas chilenas los impuestos diferidos en sus estados financieros. [How Chilean public limited companies disclose deferred taxes in their financial statements]. Revista $\quad$ Activos, 17(1), $115 \quad$ - 138. https://doi.org/10.15332/25005278.5396

Mena-Trujillo, R. P., Narváez-Zurita, C. I., \& Erazo-Álvarez, J. C. (2019). La planificación tributaria como herramienta financiera para la optimización de los recursos empresariales. [Tax planning as a financial tool for optimizing business resources]. Visionario Digital, 3(2.1.),

6-34. https://doi.org/10.33262/visionariodigital.v3i2.1.540

Oyewo, B. (2020). Outcomes of interaction between organizational characteristics and management accounting practice on corporate sustainability: the global management accounting principles (GMAP) approach. Journal of Sustainable Finance \& Investment, $1(1), \quad 1 \quad$ - 35. https://doi.org/10.1080/20430795.2020.1738141

Paredes, C, \& Deás-Albuerne, J. (2019). Aplicación de la Norma Internacional de Contabilidad n.o 12 «Impuesto diferido». [Adoption of the International Accounting Standard 12 "Deferred Tax"]. Cofin Habana, 13(1), e12.

Presidencia de la República. (2018). Registro Oficial Suplemento número 209. Reglamento para la aplicación de la Ley de Régimen Tributario Interno. [Regulation for the application of the Internal Tax Regime Law]. Recuperado de https://n9.cl/b3g0

Presidencia de la República. (2018). Suplemento, registro oficial número 392. Reglamento para la aplicación de la Ley Orgánica para el Fomento Productivo, Atracción de Inversiones, Generación de Empleo y Estabilidad. [Regulation for the implementation of the Organic Law for Productive Promotion, Investment Attraction, Employment Ge]. Recuperado de https://cutt.ly/wiQiwMO

Presidencia de la República. (2019). Registro Oficial No.31. Ley de Régimen Tributario Interno. [Official Registry No.31. Internal Tax Regime Law]. Recuperado de https://n9.cl/mkr3y 
Quizhpi-Barbecho, R., Narváez-Zurita, C., \& Erazo-Álvarez, J. (2019). La gestión contable de las empresas comerciales en el marco de las NIIF para PYMES. [Accounting management of commercial companies under the IFRS for SMEs]. Revista Arbitrada Interdisciplinaria Koinonía, 4(2), 265-298. http://dx.doi.org/10.35381/r.k.v4i2.475

Ruiz-Armijos, A., Narváez-Zurita, C., \& Erazo-Álvarez, J. (2019). Tratamiento del impuesto diferido a partir de la valuación de activos biológicos bajo NIIF en la industria camaronera del Ecuador. [Deferred tax treatment based on the valuation of biological assets under IFRS in the shrimp industry of Ecuador]. Revista Arbitrada Interdisciplinaria Koinonía, 4(2), 299 - 320. http://dx.doi.org/10.35381/r.k.v4i2.476

Servicio de Rentas Internas. (2015). Circular NAC-DGECCGC15-00000012. Ley de Régimen Tributario Interno. [Circular NAC-DGECCGC15-00000012. Internal Tax Regime Law]. Recuperado de https://cutt.ly/0o389FC

Soto, C. A., García, M. C., \& Grisales, J. A. (2018). Impuesto diferido de la medición posterior al reconocimiento de las propiedades, planta y equipo. [Deferred tax on post-recognition measurement of property, plant and equipment]. Entramado, 14(1), 128-144. https://doi.org/10.18041/entramado.2018v14n1.27111

Trofimova, L., Prodanova, N., Nudel, S., Dikikh, V., \& Savina, N. (2020). The essence of temporary differences under the conditions of changes in RSA 18/02 and convergence with IAS 12 "Income taxes" and their impact on the financial statements. Amazonia Investiga, 9(26),

415-422. https://doi.org/10.34069/Al/2020.26.02.48

Tucker, B., \& Scully, G. (2020). Fun while it lasted: Executive MBA student perceptions of the value of academic research. Accounting Education, 29(3), 263-290. https://doi.org/10.1080/09639284.2020.1736590

Vargas, C., \& Peña, A. (2017). La globalización económica y contable: Su incidencia sobre la fiscalidad en Colombia. [Economic and accounting globalization: Its impact on taxation in Colombia]. Actualidad contable FACES, 20(35), 126 - 154.

(C)2020 por los autores. Este artículo es de acceso abierto y distribuido según los términos y condiciones de la licencia Creative Commons Atribución-NoComercial-Compartirlgual 4.0 Internacional (CC BY-NC-SA 4.0)

(https://creativecommons.org/licenses/by-nc-sa/4.0/ 